\title{
Clinical Efficacy and Safety of Atorvastatin for Chronic Subdural Hematoma: A Randomized Controlled Trial
}

\author{
KUN YANG, MING QIU*, HAN ZHAO, Z. LIU AND LIMIN ZHENG \\ Department Neurosurgery, Taian City Central Hospital, Taian City, Shandong Province, China
}

Yang et al.: Clinical efficacy and safety of atorvastatin

\begin{abstract}
The aim of the present investigation was to observe the efficacy and safety of atorvastatin on chronic subdural hematoma patients. A total of 58 chronic subdural hematoma patients were recruited in this study, randomly divided into the control group and the atorvastatin treatment group (2 patients withdrew; $\mathrm{n}=28$ each group). Before and after $24 \mathrm{w}$ of treatment, the clinical outcome in patients was evaluated. The activities of daily living and neurological deficit scoring standard scores were assessed at 4, 8, 12 and $24 \mathrm{w}$ after treatment. Levels of clinical biochemical indicators, serum high-sensitivity C-reactive protein, interleukin 6, matrix metalloproteinase 9 and tumour necrosis factor $\alpha$ were presented. Furthermore, hematoma volume was detected, which was used to assess the speed and degree of hematoma absorption. The adverse reactions and recurrence rates were recorded. The total effective rate of the atorvastatin treatment group was significantly higher than that of the control group $(p=0.024)$. Following treatment, the activities of daily living score $(98.3 \pm 9.64)$ of the treatment group was significantly higher than that of the control group $(83.8 \pm 6.85 ; p=0.013)$. Moreover, the neurological deficit scoring standard score (13.8 \pm 3.89$)$ of the atorvastatin-treated group was distinctly lower than that of the control group $(20.1 \pm 4.67 ; p=0.021)$. On w 24 of treatment, compared to the control group, the serum high-sensitivity C-reactive protein, interleukin 6, matrix metalloproteinase 9 and tumour necrosis factor $\alpha$ levels in the treatment group were significantly reduced. Hematoma volume of the treatment group was significantly absorbed compared to the control group $(\mathbf{p}=\mathbf{0 . 0 0 8})$. There was no statistical significance in the incidence of various adverse reactions between the two groups. The recurrence rate of the control group $(3.60 \%)$ was significantly higher than that of the atorvastatin treatment group $(25.00 \% ; \mathbf{p}<0.05)$. These findings demonstrated that atorvastatin could reduce inflammatory response, promote hematoma absorption, protect neurological status and reduce the recrudescence rate for patients with chronic subdural hematoma.
\end{abstract}

Key words: Atorvastatin, chronic subdural hematoma, hematoma absorption, inflammatory response, recrudescence

Chronic subdural hematoma $(\mathrm{CSDH})$ is increasing year by year due to the aging population ${ }^{[1]}$. The incidence of $\mathrm{CSDH}$ in the population is 1.7-18/100 000 and the incidence in the elderly over 65 is higher, reaching $58 / 100000^{[2]}$. It is expected to reach 121.4/100 000 in 2030. More than $80 \%$ of $\mathrm{CSDH}$ patients have a history of craniocerebraltrauma ${ }^{[3]}$. Clinically, CSDH mainly manifests as severe headache, vomiting caused by increased intracranial pressure, accompanied by optic nerve papilledema ${ }^{[4]}$. Moreover, some patients exhibit mental symptoms such as dementia, apathy, slow response and local brain-derived symptoms. The current treatment for $\mathrm{CSDH}$ is to remove the hematoma by surgery, but the risk of postoperative complications and recurrence is higher ${ }^{[5]}$. Therefore, there is an urgent need to explore safer and more effective non-surgical treatment.

Hematoma adventitia neovascularization and inflammatory response are the main factors for $\mathrm{CSDH}$ pathogenesis $^{[6]}$. Furthermore, inflammatory response is closely related to the recurrence of CSDH after surgery ${ }^{[7]}$. More and more studies have found that increase in the hematoma absorption rate of subdural hematoma could reduce the recurrence rate of $\mathrm{CSDH}^{[8]}$. In recent years, statins have been used to regulate angiogenesis and repair nerve damage ${ }^{[9,10]}$. Atorvastatin can activate the Akt and Notch pathways ${ }^{[11,12]}$, thereby promote angiogenesis and functional blood vessel formation ${ }^{[13]}$. The purpose of this study was to observe the effects of atorvastatin on clinical efficacy, neurological function

*Address for correspondence

E-mail: m16531754010_1@163.com

Special Issue 5, 2020 
evaluation, serum proinflammatory factor levels, hematoma absorption, adverse reaction and recurrence rate in patients with $\mathrm{CSDH}$. This study could provide novel insights into the conservative treatment of $\mathrm{CSDH}$.

\section{MATERIALS AND METHODS}

\section{Patients:}

In this study, a total of 58 patients with CSDH admitted to Tai'an Central Hospital from October 2017 to December 2018 were selected as study subjects. The diagnostic criteria for CSDH were as follows, elderly people had symptoms of chronic high intracranial pressure, intellectual or mental abnormalities, especially with a history of mild head trauma for more than $3 \mathrm{w}$, who should be considered the possibility of CSDH; if computed tomography (CT) examination showed crescent or half-moon-shaped low-density or equaldensity shadows on the brain surface, CSDH should be diagnosed. After confirmation of enrolment, these patients were randomly divided into a control group $(n=28)$ and an atorvastatin treatment group $(n=30)$. Age, gender, initial symptoms at admission, neurological function score and other basic conditions were recorded. Patients in the atorvastatin treatment group were given a prescribed course of atorvastatin, while those in the control group were treated with conventional nutritional neuropharmacology. Complications and adverse reactions in patients in the control group and the atorvastatin treatment groups were recorded. In the atorvastatin treatment group, 2 patients withdrew from the trial. All patients signed written informed consent. The study was approved by the Ethics Committee of Tai'an Central Hospital (2020-09).

\section{Inclusion and exclusion criteria:}

The inclusion criteria were as follows, patients who met the diagnostic criteria of CSDH in neurosurgery; head CT or magnetic resonance imaging (MRI) showed hematoma thickness $<5 \mathrm{~mm}$, no significant spaceoccupying effect, and midline shift $<1 \mathrm{~cm}$; there was no risk of cerebral hernia, and conservative treatment was feasible; patients who had undergone surgery or refused surgery on the subdural hematoma in CSDH. The exclusion criteria were, history of diabetes; those who had undergone major surgery, severe trauma, or stroke in the past $6 \mathrm{mo}$; those with cerebral hernias that were extremely dangerous and required immediate surgery; mental disorders; severe liver and kidney dysfunction; heart failure (grade $\alpha$ or above) or hypertension (grade $\beta$ or above); intracranial hypertension; those who had been taking aspirin or atorvastatin for a long time; allergy to atorvastatin and pregnant women.

\section{Study design:}

In this study, two numbers 0 and 1 were randomly generated by computer and distributed. All CSDH patients were assigned into 2 groups. After randomization, 30 cases were assigned in the atorvastatin treatment group and 28 cases in the control group. After CSDH patients were admitted to the hospital, the medical history collection, physical examination and basic condition analysis of the 2 groups of patients including gender, age, and average arterial pressure were recorded. Patients in the control group were treated with conventional nutritional neuropharmaceuticals. Furthermore, attention was paid to bed treatment, limited use of dehydrating agents, correction of water and electrolyte disorders, protection of gastric mucosa and improvement of heart function. Patients in the atorvastatin treatment group received $20 \mathrm{mg}$ of atorvastatin daily after dinner on the basis of the treatment in the control group. Atorvastatin was purchased from Pfizer Pharmaceutical Co., Ltd. (Approval number: National Pharmaceutical Standard H20051408, specification: $20 \mathrm{mg} / \mathrm{d}$ ). The course of treatment was 2 mo. During the treatment, if a treatment emergency occurred, it should be handled in accordance with the principles of neurosurgery emergency treatment.

\section{Observation indices:}

At 4, 8, 12 and $24 \mathrm{w}$ after treatment, the effectiveness and safety of the treatment was assessed. The effectiveness was evaluated as follows, evaluation according to clinical effectiveness: a. hematoma elimination $\geq 99 \%$, as cured; b. hematoma elimination $50-98 \%$, as significant effectiveness; c. hematoma elimination 30-39 \%, as effectiveness; $d$. hematoma elimination $\leq 29 \%$, as ineffectiveness. Herein, total effectiveness $=\quad$ cure + significanteffectiveness + effectiveness, and total effectiveness rate $=$ (cure+ significant effectiveness + effectiveness)/total number of samples. Evaluation of the activities of daily living (ADL) and neurological deficit scoring standard (CSS).Evaluation of some characteristic biochemical indices before and after treatment. Serum highsensitivity C-reactive protein (hs-CRP) and matrix metalloproteinase 9 (MMP-9), Interleukin 6 (IL-6) and tumour necrosis factor $\alpha$ (TNF- $\alpha$ ) levels were measured. Evaluation according to the hematoma volume before 
and after treatment. Safety was assessed as follows, the changes of 3 routines of hematuria and stool, liver and kidney function, blood coagulation indices and electrocardiogram before and after treatment were recorded and patient's complications and adverse reactions (ADRs) during the treatment process were recorded.

\section{Statistical analysis:}

All data were analysed using SPSS 23.0 software. The measurement data were expressed as mean \pm standarddeviation (SD), which were assessed for normality test and homogeneity test of variance. Analysis of variance was used to compare the baseline data of the 2 groups before treatment. Before and after treatment, the paired $t$ test was used for comparison. Analysis of variance was used for comparison between groups after treatment. $\mathrm{P}<0.05$ indicated that the difference was statistically significant.

\section{RESULTS AND DISCUSSION}

In this study, 58 patients with CSDH admitted to Tai'an Central Hospital from October 2017 to December 2018 were enrolled. The initial randomization was as follows, atorvastatin treatment group $(\mathrm{n}=30)$; control group $(\mathrm{n}=28)$. Two patients in the atorvastatin treatment group withdrew, while the control group had no withdrawal. Overall, the withdrawal rate was $3.45 \%$. Finally, a total of 56 cases were included in the study, 28 cases in each group. There were 30 males and 26 females, aged 43 $-76 \mathrm{y}$, with an average age of $60.16 \pm 7.89 \mathrm{y}$. After collecting the medical history, the causes of injury were found to be due to fall and injury $(n=19)$, fall injury $(n=17)$, shock injury $(n=11)$ and traffic accident $(n=9)$. There were 42 cases with one side CSDH and 14 cases with CSDH on both sides.

Compared to before treatment, the number of cured, significant effectiveness and effectiveness patients in the atorvastatin group reached 15, 6 and 4, with a total effective rate of $89.29 \%$. Furthermore, the number of cured, significant effectiveness and effectiveness patients in the control group were 8,6 , and 5, while 9 were ineffectiveness, with a total effective rate of $67.86 \%$. Table 1 shows the clinical efficacy of the atorvastatin group and the control group. Total effective rate of atorvastatin group was significantly higher than that of the control group.

At admission, the ADL and CSS scores of CSDH patients in the atorvastatin group and control group were assessed. The results showed that there was no statistical difference between the ADL and CSS scores of the two groups of CSDH patients (Table 2). After basic care and treatment, at the end of treatment, the ADL score of CSDH patients in the control group reached $83.8 \pm 6.85$, while that of the $\mathrm{CSDH}$ patients in the atorvastatin group reached $98.3 \pm 9.64$ (Table 2). There were significant statistical differences in the ADL scores between the two groups. Furthermore, at the end of treatment, the CSS score of CSDH patients in the control group was $20.1 \pm 4.67$, while the CSS score of those in the atorvastatin treatment group was $13.8 \pm 3.89$ (Table 2). A significant difference in CSS scores was found between the groups. From the 4 th $w$ of treatment, the ADL scores of CSDH patients in the atorvastatin treatment group and the control group continued to increase, indicating that the patients' self-care ability

TABLE 1: COMPARISON OF CLINICAL EFFICACIES BETWEEN TWO GROUPS

\begin{tabular}{lccccc}
\hline Group & Cure & $\begin{array}{c}\text { Significant } \\
\text { effectiveness }\end{array}$ & Effectiveness & Ineffectiveness & $\begin{array}{c}\text { Total effectiveness } \\
\text { rate }\end{array}$ \\
\hline Atorvastatin $(n=28)$ & $15(53.57)$ & $6(21.43)$ & $4(14.29)$ & $3(10.71)$ & $25(89.29)$ \\
Control $(n=28)$ & $8(28.57)$ & $6(21.43)$ & $5(17.86)$ & $9(32.14)$ & $19(67.86)$ \\
$X^{2} / P$ value & & & & $6.325 / 0.024$ \\
\hline
\end{tabular}

TABLE 2: COMPARISON OF ADL AND CSS SCORES BETWEEN TWO GROUPS OF CSDH PATIENTS BEFORE AND AFTER TREATMENT

\begin{tabular}{lcccccc}
\hline Scores & Groups & $\begin{array}{c}\text { Before } \\
\text { treatment }\end{array}$ & $4 w$ & $8 w$ & $12 w$ & $24 w$ \\
\hline ADL & Atorvastatin $(n=28)$ & $70.4 \pm 5.20$ & $74.6 \pm 6.10$ & $80.4 \pm 6.32$ & $89.5 \pm 7.43$ & $98.3 \pm 9.64$ \\
& Control $(\mathrm{n}=28)$ & $71.2 \pm 5.18$ & $72.4 \pm 6.08$ & $76.1 \pm 4.90$ & $81.7 \pm 5.47$ & $83.8 \pm 6.85$ \\
& t/P value & $0.176 / 0.678$ & $3.764 / 0.037$ & $3.002 / 0.028$ & $3.228 / 0.045$ & $4.387 / 0.013$ \\
& Atorvastatin $(\mathrm{n}=28)$ & $28.7 \pm 5.32$ & $24.3 \pm 5.01$ & $20.9 \pm 4.97$ & $16.4 \pm 4.65$ & $13.8 \pm 3.89$ \\
CSS & Control $(\mathrm{n}=28)$ & $28.9 \pm 5.43$ & $26.6 \pm 5.52$ & $23.9 \pm 5.02$ & $22.7 \pm 4.92$ & $20.1 \pm 4.67$ \\
& t/P value & $0.100 / 0.873$ & $2.746 / 0.047$ & $3.002 / 0.038$ & $5.203 / 0.036$ & $9.307 / 0.021$ \\
\hline
\end{tabular}


continued to improve (Table 2). However, the self care ability of the patients in the atorvastatin group was significantly improved more than the control group. Furthermore, from w 4, the CSS scores of patients in the two groups continued to decrease, suggesting that the patients' neurological status continued to improve (Table 2). But the neurological status of the patients in the atorvastatin group was distinctly ameliorated compared to that of the control groups.

Before treatment, there was no statistical difference in serum hs-CRP, IL-6, MMP-9 and TNF- $\alpha$ level between the atorvastatin and control groups (Table 3). From w 4 of treatment, the levels of serum hs-CRP, IL- 6 and MMP-9 for CSDH patients in the atorvastatin group were significantly higher than those in the control group and these increased in a time-dependent manner (Table 3). On w 14, serum TNF- $\alpha$ levels were significantly lower in the atorvastatin group than the control group. Throughout the course of treatment the levels of serum hs-CRP, IL-6, MMP-9 and TNF- $\alpha$ of $\mathrm{CSDH}$ patients in the control group remained stable but gradually decreased over time, but the extent of reduction was lower than that of the atorvastatin group. Therefore, the inflammation of CSDH patients in the atorvastatin group appeared to be better controlled.
Before treatment, there was no significant difference in hematoma volume between the atorvastatin-treated group and the control group of CSDH patients (Table 4). On w 4 of treatment, compared to the control group, the hematoma volume of CSDH patients in the atorvastatin group was significantly reduced. But because the treatment cycle was not long enough on $\mathrm{w} 4$, there was no significant difference in the volume of hematoma in CSDH patients between the atorvastatin group and the control group. Starting from w 8 of treatment, the reduction of hematoma in $\mathrm{CSDH}$ patients in the atorvastatin group was significantly greater compared to that in the control group (Table 4). On the w 24 of treatment, the hematoma in 19 cases of CSDH patients in the atorvastatin group were completely absorbed, in 5 cases it was moderately absorbed while 2 cases were operated and in 4 cases it was lightly absorbed. Whereas in the control group, in 2 cases it was completely absorbed, in 2 cases moderately absorbed while 18 cases were operated and in 6 cases it was mildly absorbed.

In this study,ADRs were monitored by examining a series of clinical indicators such as 3 routines of blood, urine and stool, liver and kidney function, blood coagulation indices, and electrocardiogram in both groups. The

TABLE 3: COMPARISON OF CLINICAL INDICES BETWEEN TWO GROUPS OF CSDH PATIENTS BEFORE AND AFTER TREATMENT

\begin{tabular}{|c|c|c|c|c|c|c|}
\hline Serum levels & Groups & $\begin{array}{c}\text { Before } \\
\text { treatment }\end{array}$ & $4 w$ & $8 w$ & $12 \mathrm{w}$ & $24 w$ \\
\hline \multirow[t]{3}{*}{ hs-CRP (mg/l) } & $\begin{array}{l}\text { Atorvastatin } \\
\quad(\mathrm{n}=28)\end{array}$ & $6.4 \pm 0.41$ & $5.8 \pm 0.37$ & $5.3 \pm 0.32$ & $4.7 \pm 0.37$ & $3.5 \pm 0.18$ \\
\hline & Control $(n=28)$ & $6.4 \pm 0.38$ & $6.2 \pm 0.46$ & $5.9 \pm 0.38$ & $5.6 \pm 0.41$ & $4.8 \pm 0.23$ \\
\hline & t/P value & $0.000 / 1.001$ & $4.398 /<0.0001$ & $6.248 /<0.0001$ & $9.145 /<0.0001$ & $12.783 /<0.0001$ \\
\hline \multirow[t]{3}{*}{ IL-6 (mg/l) } & $\begin{array}{l}\text { Atorvastatin } \\
\quad(n=28)\end{array}$ & $27.1 \pm 10.02$ & $26.1 \pm 8.02$ & $24.6 \pm 5.65$ & $22.8 \pm 5.21$ & $19.0 \pm 4.57$ \\
\hline & Control $(n=28)$ & $27.1 \pm 9.89$ & $26.8 \pm 9.21$ & $25.7 \pm 7.79$ & $24.3 \pm 6.38$ & $21.9 \pm 6.34$ \\
\hline & t/P value & $0.260 / 0.973$ & $1.398 / 0.029$ & $3.235 / 0.024$ & $5.345 / 0.015$ & $7.783 / 0.012$ \\
\hline \multirow[t]{3}{*}{ MMP-9 $(\mu \mathrm{g} / \mathrm{l})$} & $\begin{array}{l}\text { Atorvastatin } \\
\quad(\mathrm{n}=28)\end{array}$ & $671.5 \pm 21.68$ & $615.3 \pm 17.96$ & $561.9 \pm 13.84$ & $450.2 \pm 9.98$ & $341.9 \pm 8.66$ \\
\hline & Control $(n=28)$ & $670.3 \pm 20.89$ & $631.1 \pm 18.73$ & $584.8 \pm 16.63$ & $499.8 \pm 13.48$ & $410.4 \pm 12.54$ \\
\hline & t/P value & $0.060 / 1.978$ & $2.398 / 0.034$ & $8.235 / 0.012$ & $12.735 / 0.008$ & $14.774 /<0.0001$ \\
\hline \multirow[t]{3}{*}{ TNF-a $(\mu \mathrm{g} / \mathrm{l})$} & $\begin{array}{l}\text { Atorvastatin } \\
\quad(\mathrm{n}=28)\end{array}$ & $44.1 \pm 10.12$ & $41.4 \pm 8.27$ & $38.5 \pm 5.84$ & $33.5 \pm 4.12$ & $28.2 \pm 2.89$ \\
\hline & Control $(n=28)$ & $44.0 \pm 10.35$ & $42.7 \pm 9.67$ & $41.1 \pm 8.97$ & $37.8 \pm 7.73$ & $34.9 \pm 6.78$ \\
\hline & $\mathrm{t} / \mathrm{P}$ value & $0.000 / 2.738$ & $1.398 / 0.062$ & $1.785 / 0.0658$ & $3.324 / 0.035$ & $3.673 / 0.033$ \\
\hline
\end{tabular}

TABLE 4: COMPARISON OF HEMATOMA VOLUME BETWEEN THE TWO GROUPS BEFORE AND AFTER TREATMENT

\begin{tabular}{lcccc}
\hline Groups & Before treatment & 4 w & 8 w & 24 w \\
\hline Atorvastatin $(n=28)$ & $20.1 \pm 4.13$ & $18.3 \pm 5.02$ & $16.4 \pm 6.13$ & $14.6 \pm 4.21$ \\
Control $(\mathrm{n}=28)$ & $19.9 \pm 4.42$ & $19.2 \pm 5.13$ & $18.5 \pm 4.79$ & $17.8 \pm 5.42$ \\
t/P value & $0.339 / 0.736$ & $0.983 / 0.102$ & $1.002 / 0.048$ & $2.839 / 0.027$ \\
\hline
\end{tabular}

Hematoma volume was expressed as mean \pm SD millilitres 
results showed that the main adverse reactions in the 2 groups of CSDH patients were nausea and vomiting, abdominal discomfort, mild elevation of transaminase, dyslipidemia and rash (Table 5). However, there was no significant difference in the incidence of ADRs between the two groups. Brain CT was used to detect recrudescence rate. The recrudescence rate of patients in the atorvastatin group (3.60\%) was distinctly lower than that in the control group $(25.00 \%)$ as shown in Table 5.

This study is a randomized controlled trial on the clinical efficacy and safety of atorvastatin for CSDH. These results demonstrated that atorvastatin could reduce inflammatory response, accelerate hematoma absorption, protect neurological status and decrease the recrudescence rate for patients with CSDH. Many studies have shown that CSDH is a local inflammatory disease $^{[7]}$. Regarding the pathophysiological changes of $\mathrm{CSDH}$, gradual conversion of chronic subdural effusion is the key to the formation of $\mathrm{CSDH}^{[14,15]}$. A series of events induced by the subdural cerebrospinal fluid makes the cell layer at the edge of the dura mater tear, thereby inducing a series of self-repair mechanisms. The local inflammation is aggravated and promotes the occurrence of hematoma. Clinical studies suggested that VEGF concentration is closely related to the hematoma exudation rate, which provided a basis for the CT imaging manifestation of hematoma ${ }^{[16]}$. Additionally, in the process of hematoma absorption in CSDH patients, VEGF concentration also indicated the speed of hematoma absorption. There are both proinflammatory and antiinflammatory response locally in $\mathrm{CSDH}^{[17]}$. Studies have found that the concentrations of proinflammatory factors such as IL-5, IL-7, TNF- $\alpha$ and bidirectional inflammatory factors such as IL-6 in hematoma fluid are significantly higher than those in peripheral blood ${ }^{[18-20]}$. Present results showed that these inflammatory factors are not only related to the formation of CSDH, but also to the mechanism of atorvastatin in the treatment of CSDH. Abnormal angiogenesis may be an important cause of CSDH. MMP-9 is a member of the matrix metalloproteinase family, which is involved in various pathological processes of central nervous system diseases ${ }^{[21-23]}$. Some studies have pointed out that MMP-9 may affect the occurrence of $\mathrm{CSDH}^{[24]}$. The results of this study showed that atorvastatin can effectively reduce the serum levels of the above proinflammatory factors in $\mathrm{CSDH}$ patients, thereby reducing the inflammatory response.

Atorvastatin is a hydroxymethylglutaryl coenzyme A reductase inhibitor. Recent studies have found that atorvastatinhas a significant therapeutic effectinaddition to preventing atherosclerosis and reducing the incidence of cardiovascular and cerebrovascular diseases ${ }^{[25-27]}$. Furthermore, it has a certain effect on stroke patients ${ }^{[28]}$. ADL scores have been an indispensable consideration and therapeutic index in the treatment of $\mathrm{CSDH}^{[29]}$. It has been reported that the recovery of nerve function is accompanied by angiogenesis ${ }^{[30]}$. In the early stage of trauma, due to the lack of angiogenesis and increased vascular permeability, the absorption rate of hematoma is slow. At this time, the intracranial height due to the space-occupying effect and the toxins produced by local metabolic disorders make the neurological deficits, thereby increasing new blood vessels and accelerating hematoma absorption. In this process, low-dose statins promote angiogenesis by activating endothelial no synthase ${ }^{[31]}$. Consistently, present data showed that atorvastatin can accelerate hematoma absorption in patients with CSDH compared to controls.

Atorvastatin treatment of $\mathrm{CSDH}$ is a comprehensive treatment as it inhibits the inflammatory response, promotes angiogenesis and improve nerve function, to produce a clinical cure. Studies have shown that atorvastatin downregulated the expression of VEGF in patients with $\mathrm{CSDH}$, thereby inhibiting the proliferation of vascular endothelial cells and forming new blood vessels, reducing the volume of hematoma, thereby reducing the recurrence rate of $\mathrm{CSDH}^{[31,32]}$. In addition, atorvastatin reduces then number of neutrophils, the concentration of inflammatory factors, induces antiinflammatory effects, and reduce the recurrence of CSDH hematoma. Consistent with previous results reported, current results indicated that atorvastatin

TABLE 5: COMPARISON OF THE INCIDENCE OF ADR AND RECRUDESCENCE

\begin{tabular}{lcccccc}
\hline Groups & \multicolumn{5}{c}{ ADR } \\
\cline { 2 - 7 } & $\begin{array}{c}\text { Nausea and } \\
\text { vomiting }\end{array}$ & $\begin{array}{c}\text { Abdominal } \\
\text { discomfort }\end{array}$ & $\begin{array}{c}\text { Increased } \\
\text { transaminase level }\end{array}$ & Dyslipidemia & Rash & Recrudescence \\
\hline Atorvastatin $(\mathrm{n}=28)$ & 1 & 3 & 1 & 0 & 2 & 1 \\
Control $(\mathrm{n}=28)$ & 2 & 2 & 1 & 2 & 3 & 7 \\
$\mathrm{X}^{2}$ & 0.783 & 1.083 & 0.789 & 0.474 & 0.272 & 3.892 \\
$\mathrm{P}$ value & $>0.05$ & $>0.05$ & $>0.05$ & $>0.05$ & $>0.05$ & $<0.05$ \\
\hline
\end{tabular}


treatment significantly reduced the risk of recurrence of $\mathrm{CSDH}$ in patients. Thus, atorvastatin treatment could inhibit the inflammatory response, promote angiogenesis and improve nerve function for $\mathrm{CSDH}$ treatment.

These findings suggested that atorvastatin could be an ideal drug for the treatment of CSDH. But due to the relatively small sample size in this study, its clinical effect still needs to be confirmed by larger clinical trials. Nevertheless, atorvastatin could effectively treat CSDH by promoting subdural hematoma absorption and improving nerve function, thereby improving the ability of self-care and quality of life. Despite patients treated with atorvastatin experienced adverse reactions such as nausea and vomiting, abdominal discomfort and rash, it is warranted as it can significantly reduce the relapse risks. Thus, for patients with CSDH with mild symptoms, timely treatment with atorvastatin could avoid the risks and complications caused by surgery. Mechanistically, atorvastatin in the treatment of CSDH is related to the regulation of inflammation and angiogenesis, which requires in-depth research.

\section{Conflict of interest:}

All authors report no conflicts of interest in this work.

\section{REFERENCES}

1. Jiang R, Zhao S, Wang R, Feng H, Zhang J, Li X, et al. Safety and efficacy of atorvastatin for chronic subdural hematoma in Chinese patients: a randomized clinical trial. JAMA Neurol 2018;75:1338-46.

2. Feghali J, Yang W, Huang J. Updates in chronic subdural hematoma: epidemiology, etiology, pathogenesis, treatment and outcome. World Neurosurg 2020.

3. Wang D, Gao C, Xu X, Chen T, Tian Y, Wei H, et al. Treatment of chronic subdural hematoma with atorvastatin combined with low-dose dexamethasone: phase II randomized proof-ofconcept clinical trial. J Neurosurg 2020:1-9.

4. He C, Xia P, Xu J, Chen L, Zhang Q. Evaluation of the efficacy of atorvastatin in the treatment for chronic subdural hematoma: a meta-analysis. Neurosurg Rev 2020;17:1-6.

5. Huang J, Li L, Zhang J, Gao C, Quan W, Tian Y, et al. Treatment of Relapsed Chronic Subdural Hematoma in Four Young Children with Atorvastatin and Low-dose Dexamethasone. Pharmacotherapy 2019;39:783-9.

6. Wei L, Lin C, Zhong M, Zhang J, Zhu G. Hemoglobin concentration may affect the effect of atorvastatin on chronic subdural hematoma after burr-hole drainage at high altitude. Front Neurosci 2020;14:503.

7. Edlmann E, Giorgi-Coll S, Whitfield PC, Carpenter KL, Hutchinson PJ. Pathophysiology of chronic subdural haematoma: inflammation, angiogenesis and implications for pharmacotherapy. J Neuroinflammation 2017;14:1-3.

8. Wang D, Li T, Tian Y, Wang S, Jin C, Wei H, et al. Effects of atorvastatin on chronic subdural hematoma: a preliminary report from three medical centers. J NeurolSci 2014;336:23742.

9. Pan HC, Yang DY, Ou YC, Ho SP, Cheng FC, Chen CJ. Neuroprotective effect of atorvastatin in an experimental model of nerve crushes injury. Neurosurgery 2010;67:376-379.

10. Kong Y, Cao XN, Zhang XH, Shi MM, Lai YY, Wang Y, et al. Atorvastatin enhances bone marrow endothelial cell function in corticosteroid-resistant immune thrombocytopenia patients. Blood 2018;131:1219-33.

11. Gu Q, Yang X, Lv J, Zhang J, Xia B, Kim JD, et al. AIBPmediated cholesterol efflux instructs hematopoietic stem and progenitor cell fate. Science 2019;363:1085-8.

12. Han F, Xiao QQ, Peng S, Che XY, Jiang LS, Shao Q, et al. Atorvastatin ameliorates LPS-induced inflammatory response by autophagy via AKT/mTORsignalling pathway. J Cell Biochem 2018;119:1604-15.

13. Quan W, Zhang Z, Li P, Tian Q, Huang J, Qian Y, et al. Role of regulatory $\mathrm{T}$ cells in atorvastatin induced absorption of chronic subdural hematoma in rats. Aging Dis 2019;10:992-1002.

14. Wang Y, Wang C, Liu Y. Chronic subdural haematoma evolving from traumatic subdural hydroma. Brain Inj 2015;29:462-5.

15. Tao Z, Lin Y, Hu M, Ding S, Li J, Qiu Y. Mechanism of subdural effusion evolves into chronic subdural hematoma: IL-8 inducing neutrophil oxidative burst. Med Hypotheses 2016;86:43-6.

16. Weigel R, Hohenstein A, Schilling L. Vascular endothelial growth factor concentration in chronic subdural hematoma fluid is related to compute tomography appearance and exudation rate. J Neurotrauma 2014;31:670-3.

17. Isaji T, Osuka K, Ohmichi Y, Ohmichi M, Naito M, Nakano $\mathrm{T}$, et al. expression of angiopoietins and angiogenic signaling pathway molecules in chronic subdural hematomas. J Neurotrauma 2020.

18. Du B, Xu J, Hu J, Zhong X, Liang J, Lei P, et al. A Clinical Study of the Intra-Neuroendoscopic Technique for the Treatment of Subacute-Chronic and Chronic Septal Subdural Hematoma. Front Neurol 2019;10:1408.

19. Stanisic M, Aasen AO, Pripp AH, Lindegaard KF, RammPettersen J, Lyngstadaas SP, et al. Local and systemic proinflammatory and anti-inflammatory cytokine patterns in patients with chronic subdural hematoma: a prospective study. Inflamm Res 2012;61:845-52.

20. Pripp AH, Stanišić M. The correlation between pro- and antiinflammatory cytokines in chronic subdural hematoma patients assessed with factor analysis. PLoS One 2014;9:e90149.

21. Montagne A, Nation DA, Sagare AP, Barisano G, Sweeney MD, Chakhoyan A, et al. APOE4 leads to blood-brain barrier dysfunction predicting cognitive decline. Nature 2020;581:716.

22. ROSARIO LV, Da Rosa BG, Goncalves TL, MATIAS DI, Freitas C, Ferrer VP, et al. Glioblastoma factors increase the migration of human brain endothelial cells in vitro by increasing MMP-9/CXCR4 Levels. Anticancer Res 2020;40:2725-37.

23. Deng Z, Zhou L, Wang Y, Liao S, Huang Y, Shan Y, et al. Astrocyte-derived VEGF increases cerebral microvascular permeability under high salt conditions. Aging (Albany NY) 2020;12.

24. Hua C, Zhao G, Feng Y, Yuan H, Song H, Bie L. Role of matrix metalloproteinase-2, matrix metalloproteinase-9, and vascular endothelial growth factor in the development of chronic subdural hematoma. J Neurotrauma 2016;33:65-70.

25. Ray KK, Nicholls SJ, Ginsberg HD, Johansson JO, Kalantar- 
Zadeh K, Kulikowski E, et al. Effect of selective BET protein inhibitor apabetalone on cardiovascular outcomes in patients with acute coronary syndrome and diabetes: Rationale, design, and baseline characteristics of the BETonMACE trial. Am Heart J 2019;217:72-83.

26. Polster SP, Stadnik A, Akers AL, Cao Y, Christoforidis GA, Fam MD, et al. Atorvastatin treatment of cavernous angiomas with symptomatic hemorrhage exploratory proof of concept (AT CASH EPOC) trial. Neurosurgery 2019;85:843-53.

27. Huang J, Gao C, Dong J, Zhang J, Jiang R. Drug treatment of chronic subdural hematoma. Expert OpinPharmacother 2020;21:435-44.

28. Guerra LC, Moreno MF, Hernández MJ, Gurruchaga PR, Camacho MC. Trends in the use of statins after ischaemic stroke: Have clinical practices changed? Neurologia 2020.

29. Jiang R, Wang D, Poon WS, Lu YC, Li XG, Zhao SG, et al. Effect of ATorvastatinOn Chronic subdural Hematoma (ATOCH): a study protocol for a randomized controlled trial. Trials 2015;16:1-9.

30. Saffari TM, Bedar M, Hundepool CA, Allen TB. The role of vascularization in nerve regeneration of nerve graft. Neural Regen Res 2020;15:1573-9.

31. Chelvanambi S, Gupta SK, Chen X, Ellis BW, Maier BF, Colbert TM, et al. HIV-Nef Protein Transfer to Endothelial Cells Requires Rac1 Activation and Leads to Endothelial Dysfunction Implications for Statin Treatment in HIV Patients. Circ Res 2019;125:805-20.

32. Li T, Wang D, Tian Y, Yu H, Wang Y, Quan W, et al. Effects of atorvastatin on the inflammation regulation and elimination of subdural hematoma in rats. J NeurolSci 2014;341:88-96.

This is an open access article distributed under the terms of the Creative Commons Attribution-NonCommercial-ShareAlike 3.0 License, which allows others to remix, tweak, and build upon the work non-commercially, as long as the author is credited and the new creations are licensed under the identical terms

This article was originally published in a special issue, "Biomedical Research in Healthcare Setting" Indian J Pharm Sci 2020:82(3)Spl issue5;86-92 